\title{
Research on the Dispatch Model of Power Systems with Wind Power and Pumped Storage Station
}

\author{
Bo Yuan, Yawu Zhang, Jin Zong, Shengyu Wu, Han Huang, and Lu Cheng
}

\begin{abstract}
Pumped storage is an effective option for solving the problems related to large scale wind power integration systems, such as peak regulation, etc. The coordinated generation dispatch for power systems with the mix generation of wind power, pumped storage and thermal power has dominant economical and environmental benefits. This paper proposed a two-stage coordinated generation dispatch model for this kind of system, which is consisted of day-ahead dispatch and hour-ahead dispatch. The model has the merits in accommodating uncertain wind power, reducing the operating reserve demand, at the same time, the proposed coordinated dispatch mode optimizes the pumped storage's operation from two aspects of the system economy and reliability. The modified IEEE-10 generator system is used to verify the effectiveness of the proposed model. The research has the guideline significances in determining the dispatch schemes for the systems with wind power and pumped storage, as well in planning pumped storage capacity.
\end{abstract}

Index Terms-Wind power, stochastic optimization, pumped storage station, unit commitment.

\section{INTRODUCTION}

In recent years, wind power installed capacity has been growing rapidly in China. Wind power generation does not consume primary energy, and is friendly to the environment, so it has high priority during generation scheduling in most countries and regions in the world [1]. However, due to the intermittent nature of wind energy, power is often delivered when it is not needed. Pumped storage technology would be able to store the excess power for later use. The joint generation scheduling of pumped storage and renewable power would support the penetration of renewable energy technologies.

Some researchers have focused on these aspects in recent years [1]-[6]. The authors in [2] have proposed to take the electrical energy from wind farms and convert it into different forms of energy for store. In [3], [4], different optimization approaches considering the requirements of storage capacity were applied to the operation of a wind-hydro pumping storage power system. In [5] the same authors proposed a similar model using multiple scenarios to describe the stochastic characteristics of wind power. In [6], the market environment was considered and a methodology is presented to obtain the optimal bids for the wind farm owners in the day-ahead market. The above researches on

Manuscript received October 14, 2015; revised January 15, 2016.

Bo Yuan, Shengyu Wu, Han Huang, and Lu Cheng are with the State Grid Energy Research Institute, China (e-mail: yuanbo_sgeri@126.com).

Yawu Zhang is with State Grid Xinyuan Company Ltd., China.

Jin Zong is with State Grid Jibei Electric power Co., Ltd Research Institute, China. pumped storage scheduling are either considering only the economic benefits of wind farm owners or aiming to smooth the joint output curve of wind farm and pumped storage. However, from the stand point of central dispatching a power system, it is more important to smooth the load curve supplied by thermal units while accommodating wind power as much as possible [7]. However, little attention has been put on this important issue.

For generation scheduling problem, two-stage decision model, i.e., stochastic programming, was widely used to deal with the uncertainty of wind power [8]-[13]. Generally speaking, multiple scenarios generated from wind power forecast results were used to model the variability and uncertainty in the system and a day-ahead UC schedule which can satisfy all the scenarios was obtained through optimization [14]-[16]. However, an important weakness of these published papers is that such a fixed day-ahead UC schedule may fail due to the great wind power uncertainty and lead to wind power spillage. The day-ahead wind power forecast error usually reaches up to $30 \%$ or higher [17], which means wind power output may differ greatly in different scenarios, and the other conventional generators may not be able to follow this rapid change due to their technical constraints such as ramping and min up/downtime constraints and this usually lead to wind curtailment Adding pumped storage can effectively avoid this situation as it can rapidly ramping and converting between pumping and generating condition. But currently there is little research on the joint generation scheduling of thermal generator, wind farm and pumped storage using two-stage decision model.

This paper aims to present a two-stage generation scheduling model considering wind power uncertainty and pumped storage. Compared to conventional two-stage generation scheduling model, a day-ahead pumped storage schedule is considered in the first stage to smooth the demand curve supplied by thermal units and this pumped storage schedule is updated in the second stage to mitigate the wind power forecast error according to real wind power scenarios. Simulations are performed on the IEEE-10 test system to verify the effectiveness of the proposed model.

\section{WIND POWER SCENARIO MODELING}

\section{A. Generation of Wind Power Scenario Sets}

The main uncertain factor affecting the generation dispatch of winder power is random fluctuations, which is difficult to predict. In order to model its random nature, wind power prediction results are be described with prediction curves and the error range with a given confidence level [8]. However, such continuous wind power 
output range is difficult to be directly used in power generation dispatch. Therefore, this paper will discrete it into a certain number of representative wind power scenarios. The specific method to generate wind power scenarios is as follows:

Wind power output is assumed to be a random variable as

$$
P_{W, R}^{t}=P_{W}^{t}+\xi_{W}^{t}
$$

Here $P_{W, R}^{t}$ represents the actual wind power output at time period $t, P_{W}^{t}$ represents the predicted wind power output at time period $t, \xi_{W}^{t}$ represents the prediction error. In this paper the distribution prediction error can be determined by statistical analysis to large amount of historical data, generally, it is approximated to be a normal distribution, namely, assuming $\xi_{W}^{t}$ is a random normal distributed variable with the expectance of 0 and the variance of $\delta_{W, t}^{2}$. Note that the modeling method is also applicable to other types of error distribution functions.

From (1) we know that $P_{W, R}^{t}$ is a random variable subject to normal distribution with the expectance of $P_{W}^{t}$ and the variance of $\delta_{W}^{t}{ }^{2}$, which is difficult to be directly applied to the dispatch model, so it is discreted into a finite number of intervals as shown in Fig. 1. The expectation of each interval is taking the median of the interval, and the probability is the integral of the probability density function with respect to this interval length. More partitions will offer higher accuracy, but also increase calculation time. This paper divides it into five intervals, namely, the wind power output per period may have five states, and the expected value in every state is $P_{w i}^{t}, i=1,2,3,4,5$, and the probability is $\pi_{i}^{t}$. Combination of wind power outputs of all the dispatch periods, namely the sequence:

$$
A=\left\{P_{w i_{A}}^{t} \mid t=1 \ldots T\right\}
$$

is a possible wind power output scenario. Its probability is:

$$
\pi=\prod_{t \in T} \pi_{i}^{t}
$$

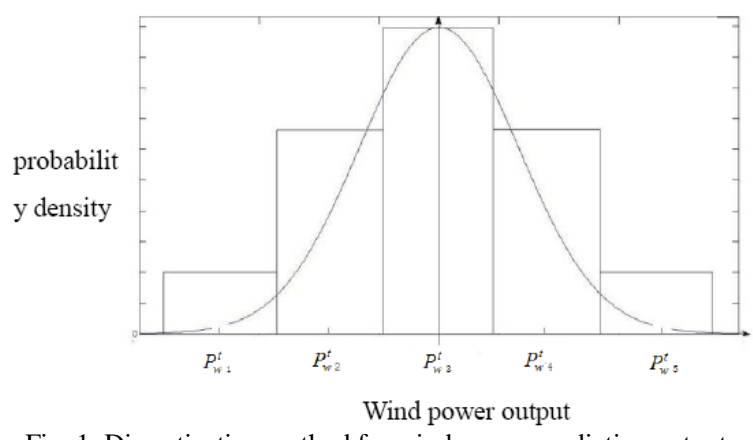

Fig. 1. Discretization method for wind power prediction output.

\section{B. Scenario Reduction}

As there are many dispatch periods, for example, for 24-period power generation dispatch, there are $5^{24}$ kinds of possible scenarios, which is computationally intractable. Hence, statistical techniques are applied to reduce the number of scenarios while retaining the essential features of the original scenario set. This paper employed the scenario reduction technique developed in [11]. (Detailed procedures are not listed here due to space limit and can be found in [12]). The reduced scenario set obtained through this process contains suitable number of scenarios for next computation.

\section{Two-Stage Generation DisPatch ModeL}

\section{A. Two-Stage Generation Dispatch Framework}

Generally speaking, the whole generation scheduling problem is formulated as a two-stage stochastic programming model here. The unit commitment and pumped storage schedule are decided based on wind power forecast results in the first stage (in this paper the first stage can be considered as day-ahead schedule). Note that these decisions do not depend on particular scenario realization and they have to satisfy all the possible wind scenarios. While in the second stage, according to each particular wind power scenario and first-stage decision, the corresponding updated pumped storage schedule and ED of thermal units are decided.

\section{B. Day-Ahead Dispatch Model}

\section{1) Objective function}

The objective function of day-ahead dispatch model contains thermal power unit start-up cost and fuel cost. The power used by pumped storage to pump water is generated by other thermal or wind units in the system, the cost has already been included as thermal fuel cost, so the only cost for pumped storage is the depreciation cost and is neglected here.

$$
\begin{gathered}
\min E=\sum_{t} \sum_{i} C_{i}^{S U}\left(1-U_{i}^{t-1}\right) U_{i}^{t}+\sum_{h} \beta_{h}\left[\sum_{t} \sum_{i} F\left(P_{G, i h}^{t}\right)\right] \\
C_{i}^{S U}=\left\{\begin{array}{c}
C_{i}^{h}, T_{i, \text { off }} \leq t_{i, \text { off }} \leq t_{i h} \\
C_{i}^{c}, t_{i, \text { off }} \geq t_{i h}
\end{array}\right. \\
t_{i h}=T_{i, \text { off }}+t_{i c} \\
F\left(P_{G, i h}^{t}\right)=a_{i} P_{G, i h}^{t}{ }^{2}+b_{i} P_{G, i h}^{t}+c_{i}
\end{gathered}
$$

where $E$ is the expected cost of the system, $U_{i}^{t}$ is the on/off condition of unit $i$ at time $t, 0$ is stop, 1 is start; $P_{G, i h}^{t}$ is the output of thermal unit $i$ in wind power scenario $h$ at time $t ; \beta_{h}$ is the possibility of occurrence of wind power scenario $h ; C_{i}^{S U}$ is the start-up cost of thermal power $i ; t_{i, o f f}$ is the continuous off time of thermal unit $i ; T_{i, o f f}$ is minimum downtime of thermal power unit $i ; t_{i c}$ is the cold start time of unit $I ; C_{i}^{h}$ is 
the hot start-up cost; $C_{i}^{c}$ is the cold start-up cost; $F\left(P_{G, i h}^{t}\right)$ is the operating cost when the thermal unit output is $P_{G, i h}^{t} ; a_{i}, b_{i}, c_{i}$ are constants.

Day-ahead dispatch model can provide the next-day generation scheduling scheme contains unit commitment $U_{i}^{t}, t=1 \ldots T, i=1 \ldots n$ of thermal units at every hour and corresponding pumped storage operating plan $P_{p, l}^{t}$.

\section{2) Constraint condition}

1) Thermal Unit Minimum up/down Time Limit

$$
\begin{gathered}
\sum_{s=t}^{t+T_{i, o n}-1} U_{i}^{s} \geq T_{i . o n}\left(U_{i}^{t}-U_{i}^{t-1}\right) \\
\sum_{s=t}^{t+T_{i, o f f}-1}\left(1-U_{i}^{s}\right) \geq T_{i . o f f}\left(U_{i}^{t-1}-U_{i}^{t}\right)
\end{gathered}
$$

where $T_{i, \text { on }}$ is the minimum operating time of unit $i$.

2) Constraint of spinning reserve

$$
\sum_{i} U_{i}^{t} P_{G, \text { imax }} \geq(1+10 \%) P_{L}^{t}
$$

3) Water dynamic balance limit

The first-stage pumped storage schedule should satisfy the water volume constraint of the upper and lower reservoir in each period

$$
\begin{gathered}
P_{P, l \min } \leq P_{P, l}^{t} \leq P_{P, l \max } \\
W_{u}^{t}=W_{u}^{t-1}+P_{P, l}^{t} \alpha_{P} \\
W_{l}^{t}=W_{l}^{t-1}+P_{P, l}^{t} \alpha_{G}
\end{gathered}
$$

$P_{P, l}^{t} \quad$ is the output of pumped storage unit $l$ at time period $t$ in day-ahead schedule; $P_{P, l \text { min }}$ is the maximum pumping output of unit $l$, which is negative; $P_{P, l \text { max }}$ is the maximum generating output of unit $l ; \alpha_{P}$ and $\alpha_{G}$ are pumping and generating conversion coefficients; $W_{u}^{t}$ is the upper reservoir capacity at time $t$; the initial capacity of the upper reservoir is $W_{u}^{0} ; W_{l}^{t}$ is the lower reservoir capacity at time $t$; the initial capacity of the lower reservoir is $W_{l}^{0}$.

4) Constraint of reservoir capacity

The pumped storage water volume should be within the maximum and minimum bounds of the upper and lower reservoirs:

$$
\begin{gathered}
W_{u \min } \leq W_{u}^{t} \leq W_{u \max } \\
W_{l \text { min }} \leq W_{l}^{t} \leq W_{l \text { max }}
\end{gathered}
$$

where $W_{u \max }, W_{l \max }$ are the maximum capacity of upper and lower reservoir, $W_{u \min }, W_{l \min }$ are the minimum.
5) Power balance constraint of every wind power scenario

$$
\sum_{i} P_{G, i h}^{t}+\sum_{j} P_{W, j h}^{t}+\sum_{l} P_{P, l h}^{t}=P_{L}^{t}
$$

where $P_{W, j h}^{t}$ is the output of wind farm $j$ at time $t$ under scenario $h ; P_{P, l h}^{t}$ is the output of pumped storage unit $l$ at time $t$ in scenario $h$.

6) Power generation limits in every wind power scenario

$$
\begin{gathered}
P_{G, i \text { min }} \leq P_{G, i h}^{t} \leq P_{G, i \text { max }} \\
P_{P, l \text { min }} \leq P_{P, l h}^{t} \leq P_{P, l \text { max }}
\end{gathered}
$$

7) The pumped storage output in every wind power scenario needs to fit with the constraint of water volume and storage capacity.

$$
\begin{aligned}
& W_{u h}^{t}=W_{u h}^{t-1}+P_{P, l h}^{t} \alpha_{P} \\
& W_{l h}^{t}=W_{l h}^{t-1}+P_{P, l h}^{t} \alpha_{G} \\
& W_{u \text { min }} \leq W_{u h}^{t} \leq W_{u \text { max }} \\
& W_{l \text { min }} \leq W_{u h}^{t} \leq W_{l \text { max }}
\end{aligned}
$$

where $W_{u h}^{t}, W_{l h}^{t}$ are upper and lower reservoir water volume at time $t$ under scenario $h$.

8) Ramping rate constraint in every wind power scenario

$$
P_{G, i h}^{t-1}-P_{D i} \leq P_{G, i h}^{t} \leq P_{G, i h}^{t-1}+P_{U i}
$$

where $P_{D i}$ is the down ramping rate of unit $I, P_{U i}$ is the up ramping rate of unit $i$.

\section{Hour-Ahead Dispatch Model}

During the hour-ahead dispatch stage, the ultra-short time wind power forecast results can be obtained, according to which the day-ahead pumped storage schedule is updated to mitigate the day-ahead wind power forecast error as much as possible while satisfying all the constraints. Then the thermal units output can be determined according to day-ahead unit commitment results and updated pumped storage schedule.

\section{1) Updating of pumped storage output in hour-ahead stage}

The objective function of updating pumped storage output is:

$$
\min D^{t}=\left|-\sum_{l} P_{p, l}^{t}-\sum_{j} P_{W, j}^{t}+\sum_{l} P_{p, l H A}^{t}+\sum_{j} P_{W, j H A}^{t}\right|
$$

where $P_{p, l}^{t}$ is the output of pump storage units in the day-ahead schedule; $P_{W, j}^{t}$ is the wind power output in the day-ahead wind power scenario which has the highest probability; $P_{W, j H A}^{t}$ is the ultra-short time wind power forecast result at time period $t$. 
Constraints include:

- Constraints of pump storage

The updated pumped storage schedule of period $t$ in each wind power scenario should also satisfy the water dynamic balance limit and reservoir limits

$$
\begin{gathered}
P_{P, l \min } \leq P_{P, l H A}^{t} \leq P_{P, l \max } \\
W_{u H A}^{t}=W_{u H A}^{t-1}+P_{P, l H A}^{t} \alpha_{P} \\
W_{l H A}^{t}=W_{l H A}^{t-1}+P_{P, l H A}^{t} \alpha_{G} \\
W_{u \min } \leq W_{u H A}^{t} \leq W_{u \max } \\
W_{l \min } \leq W_{l H A}^{t} \leq W_{l \max }
\end{gathered}
$$

where $W_{u H A}^{t}$ and $W_{l H A}^{t}$ are respectively the amount of water in the upper and lower reservoir at time period $t$ after the update of the pump storage schedule; $W_{u H A}^{t-1}$ and $W_{l H A}^{t-1}$ are respectively the water volume in the upper and lower reservoir in time period $t-1$ after the update of the pump storage schedule.

The update is based on the first stage pumped storage schedule. When the pumped storage schedule is updated in one period, the real wind power is unavailable for the rest periods, therefore the day-ahead schedule is assumed to be carried out for the rest periods, and arbitrarily regulating during one period may lead to the violation of reservoir volume constraints for the following periods. In other words, the update during time period $t$ should be on the premise of satisfying water dynamic balance limit and reservoir limits if the first-stage pumped storage schedule is carried out during the remaining periods, which means for $t^{\prime}=t+1, t+2, \ldots, T$, the following constraints should be satisfied:

$$
\begin{aligned}
& W_{u H A}^{t^{\prime}}=W_{u H A}^{t^{\prime}-1}+P_{P, l}^{t^{\prime}} \alpha_{P} \\
& W_{l H A}^{t^{\prime}}=W_{l H A}^{t^{\prime}-1}+P_{P, l}^{t^{\prime}} \alpha_{G} \\
& W_{u \min } \leq W_{u H A}^{t^{\prime}} \leq W_{u \max } \\
& W_{l \min } \leq W_{l H A}^{t^{\prime}} \leq W_{l \max }
\end{aligned}
$$

$P_{P, l}^{t^{\prime}}$ is the output of pump storage unit $l$ in the day-ahead plan at time period $t^{\prime} ; W_{u H A}^{t^{\prime}}$ is the updated output of pump storage unit $l$ at time period $t^{\prime}$.

2) Thermal units output in hour-ahead stage

The objective function is

$$
\min C^{t}=\sum_{i} F\left(P_{G, i H A}^{t}\right)
$$

Constraints include:
- The constraint of power balance

$$
\sum_{i} U_{i}^{t} P_{G, i H A}^{t}+\sum_{j} P_{W, j H A}^{t}+\sum_{l} P_{P, l H A}^{t}=P_{L}^{t}
$$

where $U_{i}^{t}$ is the on/off state of unit $i$, which is already known during the hour-ahead stage; $P_{G, i H A}^{t}$ is the output of thermal unit $i$ at time period $t$.

- The constraint of units' output

$$
P_{G, i \min } \leq U_{i}^{t} P_{G, i H A}^{t} \leq P_{G, i \max }
$$

\section{CASE Study}

\section{A. System Parameter}

In order to validate the effectiveness of the proposed model, simulation is performed on the IEEE-10 unit standard test system. The parameters of load and thermal units can be found in [17]. $250 \mathrm{MW}$ wind turbines and a $4 \times 50 \mathrm{MW}$ pumped storage station are added to the system. The parameters of the pumped storage units can be found in [18].

The binary particle swarm optimization (BPSO) is employed in this paper to solve the proposed model. PSO is a smart algorithm to solve optimization problem by sharing the information among the group and reflecting individual's own experience to enhance the individual searching strategies and finally optimizes a problem solution. The detailed formula of BPSO cannot be listed here due to the space limit of this paper, but they can be found in [13].

The parameters of the binary PSO are as follows: maximum iteration number $k_{\max }=30$; the initial particle population size is 30 ; acceleration constants $c_{1}=c_{2}=2$; inertial weight $\omega_{\max }=1.2, \omega_{\min }=0.7$.

Wind power scenarios are generated and reduced using the method proposed in Section II. The results shown in Fig. 2 , each curve in the figure represents a power wind scenario.

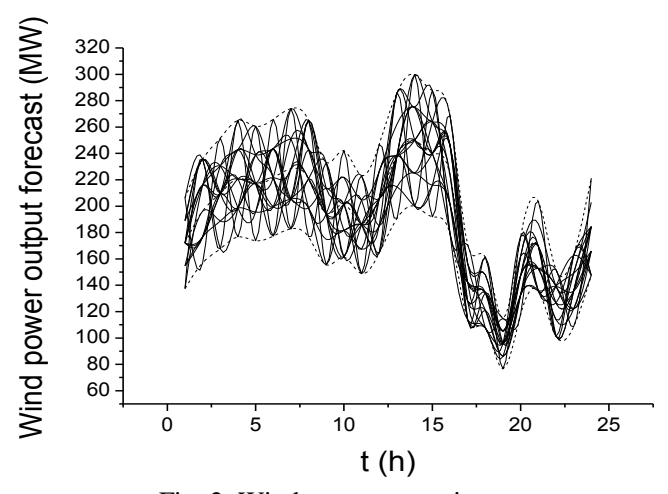

Fig. 2. Wind power scenario set.

\section{B. Evaluation Index}

In order to evaluate the effects of the proposed generation scheduling approach, this paper, besides conventional operating costs evaluation index, defines three other evaluation indexes: net load factor $\alpha$, net peak difference $\beta$, and net load standard deviation $\gamma$, specific calculation methods are as follows: 


$$
\begin{gathered}
\alpha=\text { mean }(P) / P_{\max } \\
\beta=P_{\max }-P_{\min } \\
\gamma=\delta(P)
\end{gathered}
$$

where $P$ is the net load, referring to the load carried by thermal units, that is, original load minus wind power and pumped storage output. $P_{\max }$ and $P_{\min }$ are the maximum and minimum value of net load; mean() stands for expectation value and $\delta$ stands for standard deviation.

\section{Result Analysis}

The simulation results of three most representative wind power scenarios are presented (Simulation results in other scenarios are not analyzed because of space limitation.). They are scenario 1, which has the maximum occurrence possibility, scenario 12 , which has the lowest standard deviation, and scenario 20, which has the highest standard deviation. The three scenarios are shown in Fig. 3. Given space limitations, this paper only gives the system performances index. Evaluation indexes obtained with wind power but no pumped storage are shown in Table I. When the system was added with pumped storage unit of $4 \times$ $50 \mathrm{MW}$, the evaluation indexes are shown in Table II.

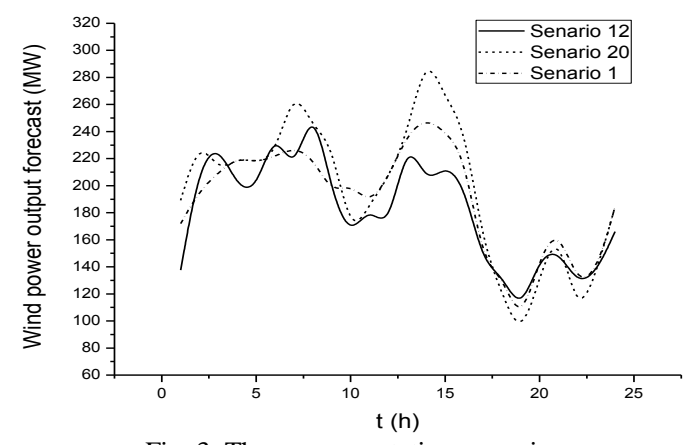

Fig. 3. Three representative scenarios.

Comparing Table I and Table II, one can see that after the system is added with pumped storage units, the expected cost changes from $\$ 546,420$ into $\$ 535,040$, decreasing by $2.1 \%$; the net load rate of wind power under wind power Scenario 1 rises from 0.7255 to 0.8100 ; net peak difference decreases from $766.22 \mathrm{MW}$ to $398.39 \mathrm{MW}$; and net load standard deviation representing net load fluctuation amplitude drops from 14.04MW to 10.68MW. Each index has improved significantly, indicating that the model can reasonably arrange pumping and generating condition of the pumped storage station.

Changes in demand for reserve capacity after hour-ahead scheduling is introduced are also analyzed. For convenience, this paper will not consider the impact of load forecasting error on operating reserve requirements. The operating reserve requirement reduction is the difference between the amount of wind power and the actual predicted results, see Table III.

Averaging the reserve demand variation in each period of each scenario in Table III, we can conclude that, the reserve demand reduces $15.70 \mathrm{MW}$ per hour in scenario 12 and drops $17.95 \mathrm{MW}$ per hour in scene 20 after introducing the hour-head dispatch stage. As we can see, the system performance increases significantly with the introduce of hour-ahead scheduling phase.

TABLE I: SYSTEM PERFORMANCE INDICES WITHOUT PSP

\begin{tabular}{cccc}
\hline Expected costs/\$ & \multicolumn{3}{c}{546420} \\
\hline Net load rate/\% & Scenario 1 & Scenario 12 & Scenario 20 \\
Difference of net peak & 0.7255 & 0.7201 & 0.7094 \\
and off-peak /MW & 766.22 & 783.41 & 801.43 \\
$\begin{array}{c}\text { Standard deviation of net } \\
\text { load } / \mathrm{MW}\end{array}$ & 14.04 & 13.45 & 14.74 \\
Actual operation costs $/ \$$ & 541150 & 557630 & 548170 \\
\hline
\end{tabular}

TABLE II: SYSTEM PERFORMANCE INDICES WITH 4×50MW PSP

\begin{tabular}{cccc}
\hline Expected costs $/ \$$ & 535040 \\
\hline Net load rate/\% & Scenario 1 & Scenario 12 & Scenario 20 \\
Difference of net peak & 0.8100 & 0.8058 & 0.8129 \\
and off-peak/ MW & 398.39 & 413.93 & 415.58 \\
$\begin{array}{c}\text { Standard deviation of net } \\
\text { load } / \text { MW }\end{array}$ & 10.68 & 10.78 & 11.01 \\
Actual operation costs/\$ & 530210 & 541560 & 538050 \\
\hline
\end{tabular}

\begin{tabular}{|c|c|c|c|c|c|}
\hline \multirow{2}{*}{$\begin{array}{c}\text { Time } \\
\text { interval }\end{array}$} & \multicolumn{2}{|c|}{ Reduction/MW } & \multirow{2}{*}{$\begin{array}{c}\text { Time } \\
\text { interval }\end{array}$} & \multicolumn{2}{|c|}{ Reduction/MW } \\
\hline & $\begin{array}{c}\text { Scenario } \\
12\end{array}$ & $\begin{array}{c}\text { Scenario } \\
20\end{array}$ & & $\begin{array}{c}\text { Scenario } \\
12\end{array}$ & $\begin{array}{c}\text { Scenario } \\
20\end{array}$ \\
\hline 1 & 17.19 & 34.38 & 13 & 0 & 41.16 \\
\hline 2 & 39.28 & 19.63 & 14 & 50 & 0 \\
\hline 3 & 0 & 20.96 & 15 & 23.96 & 50 \\
\hline 4 & 11.35 & 22.07 & 16 & 0 & 23.86 \\
\hline 5 & 0 & 21.72 & 17 & 14.09 & 22.77 \\
\hline 6 & 0 & 22.18 & 18 & 13.45 & 0 \\
\hline 7 & 34.31 & 22.84 & 19 & 9.88 & 0 \\
\hline 8 & 22.09 & 44.18 & 20 & 14.46 & 9.55 \\
\hline 9 & 38.82 & 0 & 21 & 0 & 0 \\
\hline 10 & 40.42 & 40.32 & 22 & 25.31 & 16.71 \\
\hline 11 & 0 & 0 & 23 & 0 & 0 \\
\hline 12 & 0 & 19.64 & 24 & 22.77 & 0 \\
\hline
\end{tabular}

TABLE III: CHANGES IN DEMAND FOR RESERVE CAPACITY AFTER HOUR-AHEAD SCHEDULING IS INTRODUCED

\section{CONCLUSION}

This paper has proposed a joint generation scheduling method aims to provide a day-ahead UC and pumped storage schedule; as well as the corresponding pumped storage schedule update strategy and thermal units ED when different wind scenarios have been realized. It has the merits in accommodating uncertain wind power and smoothing the demand curve to be supplied by conventional units in smart grids. Case studies have been presented on a 10-generator test system to validate the proposed model. The expected operating cost, net load demand and net load duration curve are used to evaluate the obtained generation schedule. Simulation results have indicated that the proposed model are effective and can significantly enhance the system performance to mitigate wind power uncertainty and forecast error.

\section{REFERENCES}

[1] State Grid Corporation of China, Specification of Dispatching and Operating Management for Wind Power, 2010.

[2] W. X. Pan, Y. W. Fan, and L. Zhu, "The optimal sizing for pumped storage system in wind farm," Transactions of China Electrotechnical Society, vol. 23, no. 3, pp. 120-124, 2008.

[3] D. Liu, Z. Z. Tan, and F. Wang, "Study on combined wind power with pump storage hydropower system," Shanghai Electric Power, no. 1, pp. 39-42, 2007

[4] J. Garcia-Gonzalez, R. M. R. de la Muela, and L. M. Santos, "Stochastic joint optimization of wind generation and pumped-storage 
units in an electricity market," IEEE Transactions on Power Systems, vol. 23, no. 2, pp. 460-468, 2008.

[5] Y. Z. Sun, J. Wu, and G. J. Li, "Dynamic economic dispatch considering wind power penetration based on wind speed forecasting and stochastic programming," Proceedings of the CSEE, vol. 29, no. 4, pp. 41-47, 2009.

[6] Z. Wei, P. Yu, and S. Hui, "Dynamic economic dispatch in wind power integrated system," Proceedings of the CSEE, vol. 29, no. 25, pp. 13-18, 2009.

[7] T. Yuan et al., "Short-term wind power output forecasting model for economic dispatch of power system incorporating large-scale wind farm," Proceedings of the CSEE, vol. 30, no. 13, pp. 23-27, 2010.

[8] State Grid Corporation of China, Specification of Wind Power Forecasting System, 2010.

[9] B. Zhang, W. Wu, and T. Y. Zheng, "Design of a multi-time scale coordinated active power dispatching system for accommodating large scale wind power penetration," Automation of Electric Power Systems, vol. 35, no. 1, pp. 1-6, 2011.

[10] A. Kusiak and Z. Zhang, "Short-horizon prediction of wind power: A data-driven approach," IEEE Transactions on Energy Conversion, vol 25, no. 4, pp. 1112-1122, 2010.

[11] H. Holger and R. Werner, "A note on scenario reduction for two-stage stochastic programs," Operations Research Letters, vol. 35, no. 6, pp.731-738, 2007.

[12] V. S. Pappala, I. Erlich, and K. Rohrig, "A stochastic model for the optimal operation of a wind-thermal power system," IEEE Transactions on Power Systems, vol. 24, no. 2, pp. 940-950, 2009.

[13] P.-H. Chen, "Pumped-storage scheduling using evolutionary particle swarm optimization," IEEE Transactions on Energy Conversion, vol. 23, no. 1, pp. 294-301, 2008.

[14] X. Yuan et al., "An improved binary particle swarm optimization for unit commitment problem," Expert Systems with Applications, vol. 36, no. 4, pp. 8049-8055, 2009.

[15] W. X. Ge, "Ramp rate constrained unit commitment by improved priority list and enhanced particle swarm optimization," in Proc. 2010 International Conference on Computational Intelligence and Software Engineering (CISE), 2010, pp. 1-8.

[16] C. Xing, Power System Steady State Analysis, 3rd ed. Beijing: China Electric Power Press, 2007.
[17] X. Shu, "Differential evolution algorithm for solving dynamic economic dispatch considering wind power penetration," North China Power Electric University, Beijing, 2010.

[18] M. T. Fan, Z. P. Zhang, and S. Y. Yang, "Economic dispatch of multi-regional power network involving pump storage plants," Power System Technology, vol. 24, no. 8, pp. 57-61, 2000.

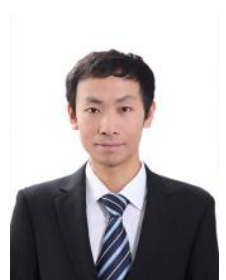

Bo Yuan was born in Henan Province, China. He received the B.S. degrees in mathematics from Nankai University in 2009, and in electrical engineering from North China Electric Power University in 2014, respectively. Currently, he is with the State Grid Energy Research Institute. His main research interest is power system reliability analysis and operation control.

Yawu Zhang is currently with the State Grid Xinyuan Company Ltd., his main research interest is the operation an planning of pumped storage station in power systems.

Jin Zong was born in Jiangxi Province, China. She received her master's degree in electrical engineering from North China Electric Power University in 2012. Currently, she is with State Grid Jibei Electric Power Co., Ltd Research Institute. Her main research interest is power system operation and distributed solar technology

Shengyu Wu was born in Jiangxi Province, China. Currently, she is with the State Grid Energy Research Institute. Her main research interest is power system planning and operation control.

Han Huang was born in Liaoning Province, China. Currently, he is with the State Grid Energy Research Institute. His main research interest is power system planning and operation control.

Lu Cheng was born in Hubei Province, China. Currently, he is with the State Grid Energy Research Institute. His main research interest is power system planning and operation control. 